\title{
AI Re-Emerging as Research in Complex Systems
}

\author{
Kemal A. Delic, Umeshwar Dayal \\ HP Technology Services Group - HP Labs \\ Hewlett-Packard Co. \\ e-mail: [ kemal.delic | umeshwar.dayal ]@hp.com
}

\begin{abstract}
The history and the future of Artificial Intelligence could be summarized into three distinctive phases: embryonic, embedded and embodied. We briefly describe early efforts in AI aiming to mimic intelligent behavior, evolving later into a set of the useful, embedded and practical technologies. We project the possible future of embodied intelligent systems, able to model and understand the environment and learn from interactions, while learning and evolving in constantly changing circumstances. We conclude with the (heretical) thought that in the future, AI should re-emerge as research in complex systems. One particular embodiment of a complex system is the Intelligent Enterprise.
\end{abstract}

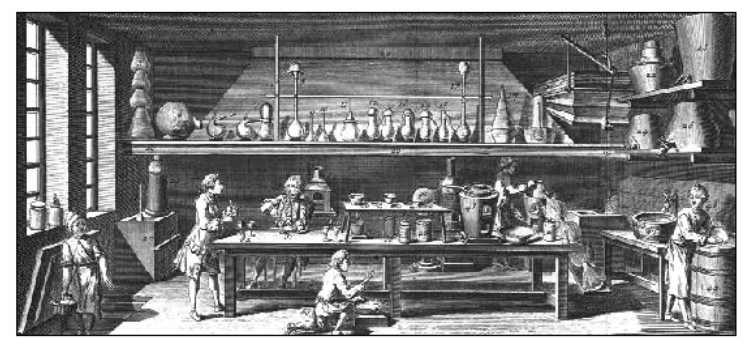

Fig. 1. AI Research as Medieval Alchemy

\section{The Early Life of AI Research}

Research in AI started with the noble objective of creating computer programs that exhibit human intelligence, promising some great achievements in a projected timeline. It has passed through several alternating cycles of brave promises and grave disappointments. Some embryonic applications in search, game playing, language understanding, expert systems, vision, robotics, and automatic programming were interesting, but stayed typically at the level of (unscalable) prototypes.

This early period spawned the development of some key technologies, much like medieval alchemist who invented important chemical compounds and processes while trying to turn lead into gold (see Fig. 1), early AI researchers developed several key technologies while chasing their field's overly ambitious goals. That period saw the development of important conceptual models, which later served as the core of AI-inspired technology developments. Several technological domains such as knowledge representation, information extraction, semantic inferencing, machine learning, probabilistic reasoning and data mining were born out of AI. 


\section{Entering the Mature Age of AI Technologies}

Paradoxically, AI has achieved success through invisible but working technologies, embedded into solutions, while disappearing from the glitzy public media and avoiding prolonged debates about the nature of intelligence. Parallel advances in IT enabled the realization of the early plans of AI communities, which were split now into several (competitive) schools of thought. It is important to mention that AI researchers also imported techniques from applied mathematics, especially from probability and statistics. In the second, mature age, toy prototypes evolved into serious technologies which evolved into realworld business systems.

Chess, go and backgammon machines able to compete against champions, global search engines capable of searching billions of web pages, embedded car and consumer device technologies, automated robotic production lines, financial screening and automated trading engines, decision support systems, space exploration voyagers, multi-player Internet games, and mobile intelligent agents are only a few notable examples of AI-inspired technologies that have attained serious deployment in consumer, business, and scientific systems. The emerging self* (management, healing, configuration) or autonomic technologies promises to spawn yet another wave of technology advances based on the early AI ideas and concepts.

\section{On the Re-Emerging Future of AI}

At present, we seem to be right now in the phase of omnipresent needs for embedded AI systems emulating intelligent behavior(s) within business and/ or consumer systems. The emergence of global, large-scale systems has brought radical technology improvements for creating, transferring and processing torrents of computer-generated data. We have already seen AI deliver on some of its early promises during its middle age (after 50 years), for example by replacing human labor with robots. Replacing human intellectual feats with machines may take another 50 years and might require a very different $\mathrm{AI}$ architecture -- for instance a hybrid one with paired silicon and wet chips bringing artificial and living matter together with corresponding/ corroborative 'computation' and 'cognition' activities (see Fig. 2).

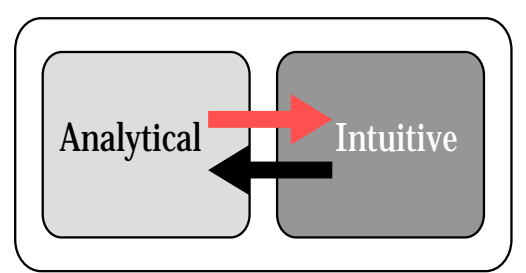

Architecture of Hybrid AI Systems (Living +Artificial)

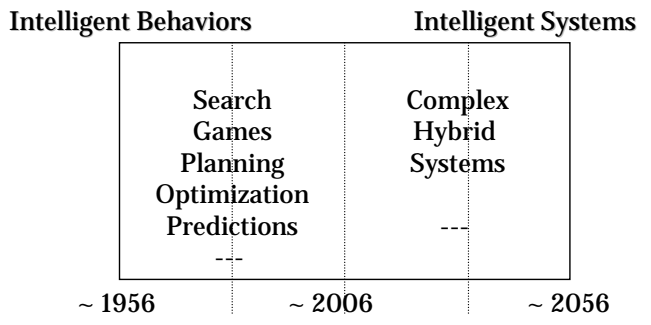

The Long View: 100 Years of AI

Fig. 2. a. Hybrid AI Systems - Embodied b. The Long View of AI Research 
We may yet witness the emergence of embodied intelligence realized as intelligent (omnipresent, dependable) systems.

By comparing the history of AI research with the history of research in complex systems (which, coincidently, also started in the early 1950's) one can conclude that both fields are exploring similar avenues. Turing and Simon are considered to be pioneers by both communities. Simply speaking, the design and architecture of man-made artificial objects and systems are very often inspired by the biological, nature-born systems, as Leonardo da Vinci has observed early in $15^{\text {th }}$ century.

Complexity, as a phenomenon, emerges typically from close interactions between the living and artificial worlds, resulting in non-linear behaviors. Such systems evolve, adapt and exhibit learning features. Thus, it would be natural and beneficial to join forces around the Science of Complex Systems. This will have (at minimum) the following benefits: enlarging and widening research, increasing the chances of creating a very large set of valuable technologies and walking away from ambitious but controversial term of "Artificial Intelligence".

We are surrounded by the host of omnipresent, complex systems (cells, markets, companies, supply networks, etc) for which elucidation of closed-loop control patterned after natural, biological systems combined with knowledge representation, learning and analytic techniques may lead the creation of largescale embodied systems. As is Intelligent Enterprise, for example, system of the high, practical value and important scientific relevance [see The Rise of The Intelligent Enterprise - http:// acm.org/ubiquity/views/k_delic_4.pdf].We believe that the future of AI will be far more successful as research in Complex Systems over the next 50 years.

\section{References}

1. Turing A.M. Computing Machinery and Intelligence (MIND, 1950).

2. Simon H. The Sciences of the Artificial (MIT Press, Boston, 1981). 